Pacific Journal of Mathematics

LATTICES OF RADICALS

Vol. 40, No. 1

September 1972 


\section{LATTICES OF RADICALS}

\section{ROBERT L. SNIDER}

Nothing first that the class of all radicals for associative rings forms a lattice under a natural ordering, we show that several important subclasses, including the class of hereditary radicals, form sublattices. We give an example showing that the special radicals do not form a sublattice even though they form a complete lattice under the same ordering.

After we have studied various lattice-theoretic properties of our main lattices, showing, in particular, that the lattice of hereditary radicals is Brouwerian, we determine the atoms of that lattice and show that it has no dual atoms by computations with free rings. We characterize pseudocomplements in the lattice of hereditary radicals and give partial results toward determining which radicals of that lattice are complemented.

We note that W. G. Leavitt [13] has remarked on some of the properties of meet and join radicals in the category of non-associative rings. We work in the category of all associative rings because of the pathology of radical theory in more general categories. (See for example [5].)

For definitions and elementary properties of radicals, see [8] or [12]. The latters $\alpha, \beta, \cdots$ will denote radicals, $\lambda, \mu, \cdots$ ordinals.

1. The Lattice of All Radicals. The results of this section are essentially a reformulation of Leavitt's work in lattice-theoretic language.

The collection of all radicals can be partially ordered by defining $\alpha \leqq \beta$ if $\alpha(R) \leqq \beta(R)$ for all ring $\mathbb{3}$. This is equivalent to the statement that all $\alpha$-radical rings are $\beta$-radical, or that all $\beta$-semisimple rings are $\alpha$-semisimple.

Let $\mathscr{R}_{\alpha}$ denote the class of all $\alpha$-radical rings, and $\mathscr{S}_{\alpha}$ the class of all $\alpha$-semisimple rings. If $\mathscr{R}$ is a radical class, let $\alpha$, denote the radical associated with $\mathscr{R}$, and if $\mathscr{S}$ is a semisimple class, let $\alpha$ denote the radical associated with $\mathscr{S}$.

Proposition 1. The class of all radicals forms a complete lattice, where for any collection $\left\{\alpha_{i}\right\}_{i \in I}$ of radicals, the semisimple class of the join is $\bigcap_{i} \mathscr{S}_{\alpha_{i}}$ and the radical class of the meet is $\bigcap_{i} \mathscr{R}_{\alpha_{i}}$.

Proof. Leavitt [13] has shown that $\bigcap_{i} \cdot \mathscr{S}_{\alpha_{i}}$ is a semisimple class and $\bigcap_{i} \cdot \mathscr{R}_{\alpha_{i}}$ is a radical class. They clearly are the join and meet 
respectively of $\left\{\alpha_{i}\right\}_{i \in I}$.

We note here that the collection of all radicals is not a set. There are at least two distinct radicals for each partition of the class of all simple rings [12], hence the collection of radicals does not have a cardinal number. Nevertheless, we refer to the collection of all radicals as a lattice.

We will denote the join of a collection of radicals $\left\{\alpha_{i}\right\}$ by $\mathrm{V} \alpha_{i}$ and the meet by $\Lambda \alpha_{i}$.

LEMmA 2. $\left(\mathrm{V} \alpha_{i}\right)(R)=R$ if and only if there exists a chain of ideals $\left\{I_{\mu}\right\}$ of $R$ such that $I_{0}=0, I_{\mu+1} / I_{\mu}$ is $\alpha_{i}$-radical for some $\alpha_{i}$, $I_{\nu}=\bigcup_{{ }^{\prime \prime}{ }_{\nu}} I_{\mu}$ if $\nu$ is a limit ordinal, and $\bigcup_{\mu} I_{\mu^{\prime}}=R$.

Proof. For the "only if" see [13]. The converse is trivial since $I_{\mu+1} / I_{\mu}$ is $\bigvee \alpha_{i}$-radical and radical classes are closed under extensions and unions.

LEMmA 3. $\left(\Lambda \alpha_{i}\right)(R)=0$ if and only if there is a descending chain of ideals $\left\{I_{\mu}\right\}$ such that $I_{0}=R, I_{n} / I_{\mu+1}$ is $\alpha_{i}$-semisimple for some $\alpha_{i}, I_{\nu}=\bigcap_{\mu<\nu} I_{\mu}$ if $\nu$ is a limit ordinal, and $\bigcap_{\mu} I_{\mu}=0$.

Proof. For the "only if" see [13]. The converse is clear.

LEMma 4. $\left(\Lambda \alpha_{i}\right)(R)=\Sigma\left\{I \leqq R: I\right.$ an ideal of $R$ and $\alpha_{i}(I)=1$ for each $\left.\alpha_{i}\right\}$.

Proof. This is immediate from Proposition 1.

2. Properties preserved by meet and join. In this section we study properties of radicals that are preserved under meet and join, thereby obtaining several sublattices of the lattice of all radicals.

Proposition 5. The class of all hereditary radicals is a complete sublattice of the lattice of all radicals.

Proof. Leavitt [13] has shown that the meet of hereditary radicals is hereditary; hence we need only show the join is hereditary. Let $\left\{\alpha_{i}\right\}$ be a collection of hereditary radicals and suppose $\left(\mathrm{V} \alpha_{i}\right)(I)=0$ where $I$ is a large ideal of a ring $R . \quad \alpha_{i}(I)=0$ for each $\alpha_{i}$. Since $\alpha_{i}$ is hereditary, $\alpha_{i}(R)=0$ by [3, theorem 3.1]. Hence $\left(\bigvee \alpha_{i}\right)(R)=0$ by Proposition 1 which implies $V \alpha_{i}$ is hereditary by [3, theorem 3.1].

E. P. Armendariz has completely determined all those hereditary radicals which are below the Baer lower radical [4]. Such a radical $\alpha$ is the lower radical determined by the zero ring $\oplus \sum_{p \in Q} Z_{p}$ where 
$Q$ is a set of primes and $Z_{p}$ is the integers modulo $p$ with the zero multiplication. From this characterization it is clear that the lattice of hereditary radicals strictly below the Baer lower radical is isomorphic to the lattice of all subsets of a countably infinite set.

Definition. A radical $\alpha$ is said to satisfy the matrix equality if $\alpha\left(R_{n}\right)=(\alpha(R))_{n}$ for all rings $R$, where $R_{n}$ denotes the $n \times n$ matrix ring with entries in $R$.

LEMma 6. If each $\alpha_{i}$ is hereditary $\left(\wedge \alpha_{i}\right)(R)=\bigcap \alpha_{i}(R)$.

Proof. Immediate.

Lemma 7. If $\alpha$ is a radical, then $\alpha\left(R_{n}\right)=I_{n}$ for some ideal $I$ of $R$ for every ring $R$.

Proof. Embed $R$ as an ideal in a ring $S$ with $1 . R_{n}$ is then an ideal of $S_{n}$ and hence $\alpha\left(R_{n}\right)$ is an ideal of $S_{n}$ [8, Theorem 47]. Since $S$ has $1, \alpha\left(R_{n}\right)=I_{n}$ for some ideal $I$ of $S$. $\alpha\left(R_{n}\right) \subseteq R_{n}$, hence $I \subseteq R$.

LEMMA 8. The following are equivalent:

(1) $\alpha$ satisfies the matrix equality

(2) $\alpha\left(R_{n}\right)=R_{n}$ if and only if $\alpha(R)=R$

(3) $\alpha\left(R_{n}\right)=0$ if and only if $\alpha(R)=0$

Proof. That (1) implies (2) and (2) implies (3) is clear. To prove (3) implies (1), we note that $\alpha(R / \alpha(R))=0$, hence

$$
\alpha\left(R_{n} /(\alpha(R))_{n}\right)=\alpha\left((R / \alpha(R))_{n}\right)=0 .
$$

Therefore, $\alpha\left(R_{n}\right) \leqq(\alpha(R))_{n}$. Also, $\alpha\left(R_{n}\right)=I_{n}$ for some ideal $I$ of $R$ by Lemma 7. Therefore, $\alpha\left((R / I)_{n}\right)=\alpha\left(R_{n} / I_{n}\right)=0$, which implies $\alpha(R / I)=0$ by (3). This implies that $I \supseteqq \alpha(R)$.

Proposition 9. The class of all radicals which satisfy the matrix equality is a complete sublattice of the lattice of all radicals.

Proof. Let $\left\{\alpha_{i}\right\}$ be a collection of radicals which satisfy the matrix equality. We must show that $\Lambda \alpha_{i}$ and $\mathrm{V} \alpha_{i}$ satisfy the matrix equality. If $\left(\Lambda \alpha_{i}\right)(R)=R$, then $R$ is $\alpha_{i}$-radical, hence $R_{n}$ is $\alpha_{i}$-radical, for each $\alpha_{i}$. Therefore $\left(\Lambda \alpha_{i}\right)\left(R_{n}\right)=R_{n}$. Similarly. $\left(\Lambda \alpha_{i}\right)\left(R_{n}\right)=R_{n}$ implies $\left(\Lambda \alpha_{i}\right)(R)=R$; hence $\Lambda \alpha_{i}$ satisfies the matrix equality by Lemma 8 if each $\alpha_{i}$ does. In the same way, $\left(\mathrm{V} \alpha_{i}\right)(R)=0$, if and only if $\left(\mathrm{V} \alpha_{i}\right)\left(R_{n}\right)=0$, and again by Lemma $8, \bigvee \alpha_{i}$ satisfies the matrix equality. 
We recall the following definition of Andrunakievic [2]. A class of rings $?$ is special if

(1) Every ring in is prime.

(2) Any nonzero ideal of a ring of $Z$ is in 2 .

(3) If $A$ is a nonzero ideal of a ring $R$ and $A$ is in $\mathscr{L}$, then $K / A^{*}$ is in $C^{2}$ where $A^{*}$ denotes the annihilator of $A$ in $K$.

A radical $\alpha$ is special if there exists a special class $\mathscr{P}$ such that $\alpha$ is the upper radical of 2 .

Let $/ /$ be a class of rings such that if $R$ is in. $/ /$ and $I$ is a nonzero ideal of $R, I$ has a nonzero homomorphic image in $\mathscr{A l}$. We will denote by $\mathscr{C}(. \mathscr{C})$ the upper radical determined by $\mathscr{C}$.

We now extend Theorem 2 of [15] to infinite unions.

Lemma 10. Let $\left\{. \mathscr{A}_{i}\right\}$ be a collection of classes of rings such that if $R$ is in $\mathscr{L}_{i_{0}}$ and $I$ is a nonzero ideal of $R, I$ has a nonzero homomorphic image in $\mathscr{A}_{i_{0}}$, then $\dddot{\ell}\left(\mathbf{U}_{2} \cdot \mathscr{A}_{i}\right)=\Lambda_{i} \not\left(\mathscr{C}_{i}\right)$.

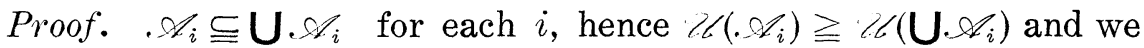

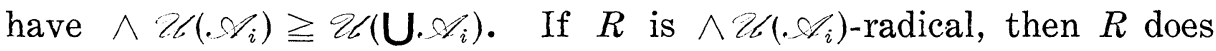
not have a nonzero homomorphic image in $\mathscr{A}_{i}$ for each $i$, and hence in $\bigcup_{i} \cdot \mathscr{L}_{i} \cdot$ Therefore $R$ is $\not \ell\left(\bigcup \cdot \mathscr{L}_{i}\right)$-radical.

Proposition 11. The meet of special radicals is special.

Proof. Let $\left\{\alpha_{i}\right\}$ be a collection of special radicals with special classes $\left\{\mathscr{O}_{i}\right\}$. Clearly $U \mathscr{C}_{i}$ is a special class. The result then follows from Lemma 10.

COROLLARY 12. The collection of all special radicals forms a complete lattice.

Although the special radicals form a lattice, it is not a sublattice of the lattice of all radicals, as the following example shows.

EXAMPLE. Let $F_{1} \subset F_{2} \subset F_{3} \subset \cdots \subset F_{n} \subset \cdots$ be a countably infinite increasing sequence of nonisomorphic fields. Let $P$ be the direct product $\prod_{i=1}^{\infty} F_{i}$ of the fields. Let $R \cong P$ be those elements $\left(f_{i}\right)$ of $P$ for which there is a finite sequence $a_{1}, \cdots a_{n}$ with $a_{i}$ in $F_{i}$ for which $f_{i}=a_{i(\bmod n)} \cdot R$ is clearly a subring of $P$. Let $\left(a_{1}, \cdots, a_{n}\right)$ denote such an element $\left(f_{i}\right)$. We now show that every prime ideal $I$ of $R$ is maximal. Consider $R / I$ and suppose $\left(a_{1}, \cdots, a_{n}\right)$ is not in $I$.

Let

$$
b_{i}=\left\{\begin{array}{lll}
a_{i}^{-1} & \text { if } & a_{i} \neq 0 \\
0 & & a_{i}=0 .
\end{array} \quad 1 \leqq i \leqq n\right.
$$


Then $\left(b_{1}, \cdots, b_{n}\right)$ is not in $I$, otherwise $\left(a_{1}, \cdots, a_{n}\right)$ is in $I$. Hence $\left(a_{1} b_{1}, \cdots, a_{n} b_{n}\right)$ is not in $I$ since $I$ is prime. $\left(a_{1} b_{1}, \cdots, a_{n} b_{n}\right)$ is idempotent and hence must be the identity modulo $I$. Therefore $R / I$ is a field and $I$ is maximal. Define $A$ and $B$ inductively as follows: Let 1 be in $A$ and 2 be in $B$. After $n$ steps, let the next $n+1$ positive integers be in $A$ and the next $n+1$ after that be in $B$. Let $A=F_{i}: i$ is in $\left.A\right\}$ and $\mathscr{S}=\left\{F_{i}: i\right.$ is in $\left.B\right\} . \mathscr{A}$ and are clearly special classes. $R$ is a subdirect sum of rings in. $\mathscr{O}$; for if

$$
J=\cap\{I: R / I \text { is in } \mathscr{Y}\}
$$

and $\left(\alpha_{i}, \cdots, A_{n}\right)$ is in $J$, we eventually have $2 n$ consecutive integers in $A$ forcing $\left(a_{1}, \cdots, \alpha_{n}\right)=0$. Similarly $R$ is a subdirect sum of rings

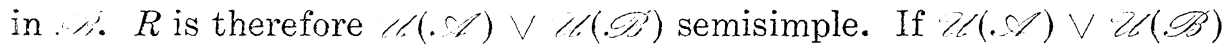
were special, $R$ would be a subdirect sum of prime $\dddot{C}(\mathscr{C}) \vee \not \mathscr{C}(\mathscr{B})$ semisimple rings [2, p. 195], hence of semisimple fields. All fields are radical, hence $t /(\mathscr{O}) \vee \measuredangle(\mathscr{B})$ is not special.

The above example gives another example of a hypernilpotent radical which is not special [18].

Let.$/$ be a class of rings. Let $\left(\mathscr{C}_{i}\right)$ denote the lower radical determined by.$/ 2[12]$. In the same vein as Lemma 10 , we have

LEMma 13. If $\left\{\mathcal{Y}_{\imath}\right\}$ are classes of rings, then

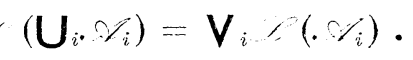

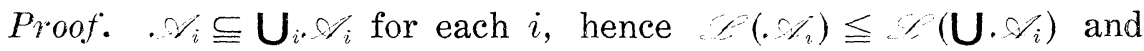

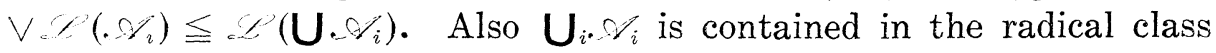
of $\mathrm{V}_{i} \mathscr{C}(\mathscr{\mathscr { C }})$, hence $\left.\mathscr{C}\left(\mathrm{U} \cdot \mathscr{C}_{i}\right) \leqq \mathrm{V}_{i} \mathscr{C}_{i}\right)$.

Let $p(x)$ and $q(x)$ be polynomials with integer coefficients. A ring $R$ is said to be $(p ; q)$ regular if for each $r$ in $R, r=p(r) s q(r)$ for some $s$ in $R$. The $(p ; q)$ regular rings form a radical class [17] for each $p$ and $q$. It is immediate from [17, Theorem 3] that the semiprime $(p ; q)$-radicals are preserved under finite meets. Musser has shown [17, Theorem 7] that the linear semiprime $(p, q)$-radicals form a lattice. It is immediate from his proof that this is a sublattice of the lattice of all radicals. We state this as

Proposition 14 (Musser). The linear semiprime ( $p ; q)$-radicals form a sublattice of the lattice of all radicals.

3. Properties of the lattices. In this section, we study elementary lattice theoretic properties of some of the lattices of the previous section. We show in particular that the lattice of all radicals is not even modular, but that the lattice of all hereditary radicals is 
Brouwerian but not dually Brouwerian.

EXAmPLE. Let $\alpha$ denote the Jacobson radical, $\beta$ the $(x ; 1)$ radical, and $\gamma$ the $(x ; x)$ radical (von Neumann regularity). It is clear that $\gamma \leqq \beta$. If $Z_{4}$ is the integers $\bmod 4$, we have $(\gamma \vee(\alpha \wedge \beta))\left(Z_{4}\right)=0$, but $((\gamma \vee \alpha) \wedge \beta)\left(Z_{4}\right)=Z_{4}$; hence $\gamma \vee(\alpha \wedge \beta) \neq(\gamma \vee \alpha) \wedge \beta$. This shows that the lattice of all radicals is not modular.

We recall [6], that a lattice $\mathscr{L}$ is Brouwerian if for any two elements $a$ and $b$ of $\mathscr{L}$, there exists a largest element $c$ such that $a \wedge c \leqq b$.

Proposition 15. The lattice of hereditary radicals forms a Brouwerian lattice.

Proof. Since the lattice of hereditary radicals is complete, we need only prove the infinite distributive law $\alpha \wedge\left(\mathbf{V}_{i} \alpha_{i}\right)=\vee\left(\alpha \wedge \alpha_{i}\right)$, [6]. In any lattice we have $\alpha \wedge\left(\vee_{i} \alpha_{i}\right) \geqq \vee(\alpha \wedge \alpha)$. Suppose now that $\left(\alpha \wedge\left(\bigvee \alpha_{i}\right)\right)(R)=R$ for some ring $R . \quad R$ is then $\bigvee \alpha_{i}$-radical and hence there exists a chain $\left\{I_{\mu}\right\}$ of ideals of $R$ satisfying the conditions. of Lemma $2 . R$ is also $\alpha$-radical. Since $\alpha$ is hereditary, we have $I_{\mu+1} / I_{\mu}$ is $\alpha$-radical, hence $\alpha \wedge \alpha_{i}$-radical, for some $\alpha_{i}$. Lemma 2 then gives us that $R$ is $\mathrm{V}_{i}\left(\alpha \wedge \alpha_{i}\right)$-radical.

CORO LARY 16. The lattice of all hereditary radicals is distributive.

CoRollary 17. If $\left\{\alpha_{i}\right\}$ is a collection of radicals and $\alpha$ is $a$ hereditary radical, then $\alpha \wedge\left(\vee \alpha_{i}\right)=\mathrm{V}_{i}\left(\alpha \wedge \alpha_{i}\right)$.

ExAmple. For each prime $p$, let $Z_{p}$ denote the integers mod $p$. Let $\mathscr{L}_{p}=\left\{Z_{p}\right\} . \mathscr{L}_{p}$ is a special class. Let $\alpha_{p}$ denote the associated special radical. $\alpha_{p}$ is hereditary [2]. $\alpha_{p}(R)=\cap\{I$ an ideal of $R$ : $\left.R / I \cong Z_{p}\right\}$ for any ring $R$ [2]. Let $\beta$ be the $(x ; x)$ radical, and let $Z$ denote the integers. Then $0=\left(\beta \vee\left(\wedge_{p} \alpha_{p}\right)\right)(Z) \neq\left(\Lambda_{p}\left(\beta \vee \alpha_{p}\right)\right)(Z)=Z$. Since the lattice of hereditary radicals is complete, this shows it is not dually Brouwerian.

The above example shows that the lattice of hereditary radicals is not complemented since complemented distributive lattices are dually Brouwerian. We now show that the Jacobson radical does not have a complement in the lattice of all radicals. If it did, all simple Jacobson radical rings (we allow rings with square 0 to be simple) would be semisimple for the complement radical. Let $E$ denote the even integers. For every ideal $(2 n)$ of $E,(2 n) /(4 n)$ is a simple Jacobson radical ring, hence $E$ is semisimple for both the Jacobson 
radical and its complement. Hence the Jacobson radical does not have a complement.

The lattice of special radicals is also Brouwerian. We first need a characterization of the join of special radicals in the lattice of special radicals. If $\alpha$ and $\beta$ are special radicals, let $\alpha \mathrm{V}_{s} \beta$ denote the join of $\alpha$ and $\beta$ in the lattice of special radicals.

Proposition 18. Let $\left\{\alpha_{i}\right\}$ be a collection of special radicals. If $\mathscr{C}_{\alpha_{i}}$ is the class of prime $\alpha_{i}$-semisimple rings, then $\bigcap_{i} \mathscr{L}_{\alpha_{i}}$ is a special class for $\mathbf{V}_{i s} \alpha_{i}$.

Proof. The $\left\{\mathscr{C}_{\alpha_{i}}\right\}$ are special classes for the $\left\{\alpha_{i}\right\}$ [2]. Clearly $\bigcap_{i \sim} \mathscr{L}_{\alpha_{i}}$ is a special class and $\mathscr{U}\left(\bigcap_{i} \mathscr{C}_{\alpha_{i}}\right) \geqq \mathrm{V}_{s} \alpha_{i}$, where $\mathscr{U}\left(\mathrm{U} \mathscr{C}_{\alpha_{i}}\right)$ denotes the upper radical of $\bigcap_{i} \mathscr{K}_{\alpha_{i}}$. If $P$ is a prime $\bigvee_{s} \alpha_{i}$-semisimple ring, then $P$ is a $\alpha_{i}$-semisimple for each $\alpha_{i}$ and hence is in $\bigcap \mathscr{L}_{\alpha_{i}}$. Since $V_{s} \alpha_{i}$ is the upper radical determined by the collection of prime $\mathbf{V}_{s} \alpha_{i}$-semisimple rings [2], (we have $\mathrm{V}_{s} \alpha_{i} \geqq \mathscr{U}\left(\boldsymbol{\bigcap}_{i} \mathscr{C}_{\alpha_{i}}\right)$.

Proposition 19. The lattice of special radicals is Brouwerian.

Proof. Again we prove the infinite distributive law $\alpha \wedge\left(\mathbf{V}_{i s} \alpha_{i}\right)=$ $\mathrm{V}_{i s}\left(\alpha \wedge \alpha_{i}\right)$, where $\alpha$ and $\alpha_{i}$ are special radicals. We always have $\alpha \wedge\left(\mathbf{V}_{i s} \alpha_{i}\right) \geqq \mathbf{V}_{i s}\left(\alpha \wedge \alpha_{i}\right)$. If $\alpha \wedge\left(\mathbf{V}_{s} \alpha_{i}\right)>\mathbf{V}_{i s}\left(\alpha \wedge \alpha_{i}\right)$, then there exists a prime $\mathbf{V}_{i s}\left(\alpha \wedge \alpha_{i}\right)$-semisimple ring $P$ which is $\alpha \wedge\left(\mathbf{V}_{s} \alpha_{i}\right)$ radical. This implies that $P$ is $\alpha \wedge \alpha_{i}$ semisimple, but $\alpha$-radical and hence $\alpha_{i}$-semisimple for each $\alpha_{i}$. We have then that $P$ is $\alpha \wedge\left(\mathbf{V}_{s} \alpha_{i}\right)$ semisimple since $P$ is $V_{i s} \alpha_{i}$-semisimple by Proposition 18.

4. Atoms. In this section, we determine all the atoms of the lattice of hereditary radicals. We also show that this lattice has no dual atoms.

Hoffman and Leavitt [9] have shown that if $\mathscr{A}$ is a hereditary class of rings, then the lower radical $\mathscr{L}(\mathscr{A})$ is hereditary.

We recall that a subring $I$ of a ring $R$ is accessible if there is a finite chain of subrings $I=I_{1} \leqq I_{2} \leqq \cdots \leqq I_{n}=R$, where $I_{i}$ is an ideal of $I_{i+1}$.

We allow simple rings to have square 0 .

Proposition 20. If $S$ is a simple ring, $\mathscr{L}(S)$ is an atom in the lattice of hereditary radicals.

Proof. Let $\alpha$ be a hereditary radical such that $0<\alpha \leqq \mathscr{L}(S)$. Let $R$ be a $\alpha$-radical ring. If $T \neq 0$ is a subdirectly irreducible factor ring of $R$ with heart $H, \alpha(H)=H$. Either $H$ is a simple ring or 
$H^{2}=0$. If $H^{2}=0$, pick $x \neq 0$ in $H$. Then the ideal of $H$ generated by $x$ is a cyclic group with zero multiplication, hence has a factor ring which is simple. In either case, we obtain a simple $\alpha$-radical ring $K$. Since $\alpha \leqq \mathscr{L}(S), K$ is $\mathscr{L}(S)$-radical and hence contains an accessible subring isomorphic to $S$ [1, lemma 1]. $K$ is simple, hence $K$ is isomorphic to $S$. This gives $\mathscr{L}(K)=\mathscr{C}(S) \leqq \alpha$.

COROLlaRY 21. If $S$ and $T$ are nonisomorphic simple rings, then $\mathscr{C}(S)=,(T)$.

COROLlary 22. Every hereditary radical contains an atom. The only atoms are of the form $\mathscr{P}(S)$ for some simple ring $S$.

Proof. If $\alpha$ is hereditary, we can obtain a $\alpha$-radical simple ring $S$ as in the proof of proposition 20. Clearly $0 \neq \varnothing(S) \leqq \alpha$.

We denote by $|S|$ the cardinality of the set $S$.

Proposition 23. The lattice of hereditary radicals contains no dual atoms.

Proof. Suppose to the contrary that $\alpha$ is a dual atom.

I. $\alpha$ is hypernilpotent. If not, $\alpha(Z)=0$, where $Z$ denotes the integers with zero multiplication since the Baer lower radical is the lower radical of $Z$ [12]. Since $\alpha$ is hereditary, we also have $\alpha\left(Q_{0}\right)=0$ where $Q_{1}$ denotes the rational numbers with zero multiplication. Let $Q$ denote the rational numbers with ordinary multiplication and $\left\langle x^{n}\right\rangle$ the ideal of the formal power series ring Q[[x]] generated by $x^{n}$. $\left\langle x^{n}\right\rangle \mid\left\langle x^{n}\right\rangle \cong Q_{0}$, which is $\alpha$-semisimple, from which it follows that $\langle x\rangle$ is a $\alpha$-semisimple. Also $\beta(\langle x\rangle)=0$, where $\beta$ is the Baer lower radical since $\langle x\rangle$ is a prime ring. We then have $(\alpha \vee \beta)\langle x\rangle=0$ and hence $\alpha \vee \beta<1$, contradicting $\alpha$ 's being a dual atom.

II. Let $F(\mathbf{W})$ denote the free ring with $\$$ generators. $F(\boldsymbol{\aleph})$ is not $\alpha$-radical for every cardinal $\$$ since every ring is a homomorphic image of a free ring and $\alpha<1$. Let $\boldsymbol{\aleph}_{i}$ be an infinite cardinal with $\alpha\left(F\left(\boldsymbol{\aleph}_{i}\right)\right)=F\left(\boldsymbol{\aleph}_{i}\right)$. Let. $/ \ell=\left\{R:|R| \leqq \boldsymbol{\aleph}_{i}\right\} . \mathscr{C}(. / /)$ is hereditary since $/ /$ is [9]. $\quad Z^{\prime}(\not /) \equiv \alpha$, and we show in III below that $F\left(\boldsymbol{\aleph}_{i}\right)$ is not $\alpha \vee P(\mathscr{C})$-radical for any $\boldsymbol{\aleph}_{j}>\boldsymbol{\aleph}_{i}$. Therefore $\alpha \vee C(/ /)<1$ and $\alpha$ is not a dual atom.

III. Let $\boldsymbol{\aleph}_{j}>\boldsymbol{\aleph}_{i}$. Let $A=\cap\left\{I\right.$ ideal of $F\left(\boldsymbol{\aleph}_{j}\right): F\left(\boldsymbol{\aleph}_{j}\right) / I$ is in and $\left.\alpha\left(F\left(\boldsymbol{W}_{3}\right) / I\right)=0\right\} . \quad A$ is a proper semiprime ideal since

$$
F\left(\boldsymbol{\aleph}_{i}\right) / \alpha\left(F\left(\boldsymbol{\aleph}_{i}\right)\right)
$$

is a homomorphic image of $F\left(\boldsymbol{\aleph}_{j}\right)$ and $\alpha$ is hypernilpotent. $A$ is 
invariant under automorphisms of $F\left(\boldsymbol{\aleph}_{j}\right)$. It follows the $\left|F\left(\boldsymbol{\aleph}_{j}\right) / A\right|=$ $\aleph_{j}$ since the generators must be distinct modulo $A$. Let $I / A$ be a nonzero ideal of $F\left(\boldsymbol{S}_{j}\right) / A$ and $i+A \neq 0$ be in $I / A$. Let $x_{\imath}$ and $x_{j}$ be generators of $F\left(\boldsymbol{\aleph}_{j}\right)$ not in the expression for $i$. There is a homomorphism $f$ of $F\left(\boldsymbol{\aleph}_{j}\right)$ onto a ring $S$ with $|S| \leqq \boldsymbol{\aleph}_{j}, \alpha(S)=0$, and $f(i) \neq 0 . f(i)$ does not annihilate $S$ since $S$ is semiprime, hence there is an $s$ in $S$ with $f(i) s \neq 0$. Clearly there is another homomorphism $h$ onto $S$ with $h(i)=f(i), h\left(x_{i}\right)=0$, and $h\left(x_{j}\right)=s$ since $F\left(\boldsymbol{\aleph}_{j}\right)$ is a free ring. We then have $h(i)\left(h\left(x_{j}\right)-h\left(x_{i}\right)\right)=f(i) s \neq 0$. It follows that $i x_{i}+A \neq i x_{j}+A$ and that $|I / A|=\boldsymbol{\aleph}_{j}$. Let $J / A$ be an accessible subring of $F\left(\boldsymbol{\aleph}_{j}\right) / A$. There is a chain $J=I_{0} \leqq I_{1} \leqq \cdots \leqq I_{n}=F\left(\boldsymbol{\aleph}_{j}\right)$ with $I_{i}$ an ideal of $I_{i, 1}$. Let $J^{*}$ be the ideal of $F\left(\boldsymbol{\aleph}_{j}\right)$ generated by $J$. Then $\left(J^{*} / A\right)^{3^{n}} \subseteq J / A$ [2] and $\left(J^{*} / A\right)^{3^{n}}$ is a nonzero ideal of $F\left(\boldsymbol{S}_{j}\right) / A$ since $\alpha$ is hypernilpotent. We then have $|J| A \mid=\boldsymbol{\aleph}_{j}$. We conclude that no accessible subring of $F\left(\boldsymbol{\aleph}_{j}\right) / A$ is in $/ /$, hence by [1, Lemma 1], $(\mathscr{C l})\left(F\left(\boldsymbol{\aleph}_{3}\right) / A\right)=0$. Also $\alpha\left(F\left(\boldsymbol{\aleph}_{j}\right) / A\right)=0$. Therefore

$$
(a \vee \nearrow(\mathscr{C}))\left(F\left(\boldsymbol{\aleph}_{j}\right) / A\right)=0,
$$

so that $(\alpha \vee,(. / /))\left(F\left(\boldsymbol{\aleph}_{j}\right) \neq F\left(\boldsymbol{\aleph}_{j}\right)\right.$.

5. Pseudocomplements. In this section, we shall study pseudocomplements in the lattice of hereditary radicals. Pseudocomplements exist since this lattice is Brouwerian [Prop. 15].

The following proposition is a restatement of Theorem 3 of [14] in a special case.

Proposition 24. Let $\mathscr{R}$ be a radical class. The largest hereditary class contained in $\mathscr{R}$ is $\mathscr{T}=\{R$ in $\mathscr{R}: I$ is an accessible subring of $R$ implies $I$ in .

Let $\alpha$ be a hereditary radical. By $\alpha^{*}$, we denote the pseudocomplement of $\alpha$ in the lattice of hereditary radicals. If $\alpha$ and $\beta$ are hereditary, let $(\alpha: \beta)$ denote the largest hereditary radical $\gamma$ such that $\gamma \wedge \beta \leqq \alpha$.

For a hereditary radical $\alpha$, Andrunakievic [2] has constructed the largest radical $\alpha^{\prime}$ such that $\alpha(R) \cap \alpha^{\prime}(R)=0$. Clearly, if $\alpha^{\prime}$ is hereditary, $\alpha^{\prime}=\alpha^{*}$. Andrunakievic has shown that when $\alpha$ is hypernilpotent or is subidempotent (every $\alpha$-radical ring is $f$-regular [7]), $\alpha^{\prime}$ is hereditary. He also has shown that $\alpha^{\prime}$ is subidempotent if $\alpha$ is hypernilpotent and conversely.

Following Andrunakievic, we call a ring $R$ strongly $\alpha$-semisimple if every homomorphic image of $R$ is $\alpha$-semisimple. The radical class 
of $\alpha^{\prime}$ is then characterized as the collection of all strongly $\alpha$-semisimple rings. $\quad \alpha^{\prime}$ is also the upper radical determined by the class of all subdirectly irreducible rings with $\alpha$-radical hearts [2, p. 183].

EXAMPLE. Let $A$ be the algebra over the rationals with basis 1 and a with $a^{2}=0$. If $\alpha$ is the radical which assigns to each ring its additive torsion subgroup, then $\alpha$ is hereditary and $\alpha^{\prime}(A)=A$. Clearly $A a$ is nonzero ideal of $A$ isomorphic to the additive group of the rationals with zero multiplication. Clearly $\alpha^{\prime}(A \alpha)=0$, hence $\alpha^{\prime}$ is not hereditary.

The above example is also a counterexample to a theorem of Michler [16]. Michler's corollary $3.20[16, \mathrm{p} .33]$ states that $\alpha^{\prime}$ must be hereditary. His error can be traced to Lemma 3.19 where he assumes that every homomorphism of an ideal $K$ of a ring $R$ is induced by a homomorphism of $R$. This error renders several of his other results false in his section $3 \mathrm{D}$.

Proposition 25. If $\alpha$ is a hereditary radical, then $R_{\alpha}{ }^{*}=\{R$ : every accessible subring of $R$ is strongly $\alpha$-semisimple\}.

For hereditary radicals $\alpha$ and $\beta$, we generalize Andrunakievic's result $[2$, p. 184] to obtain the largest radical $\gamma$ such that

$$
\gamma(R) \cap \beta(R) \leqq \alpha(R)
$$

for every ring $R$.

THEORE in 26. Let $\alpha$ and $\beta$ be hereditary radicals. If $\mathscr{R}=$ $\{R: \beta(R / I) \subseteq \alpha(R / I)$ for every ideal of $R\}$, then $\mathscr{R}$ is a radical class and $\gamma=\gamma_{\mathscr{B}}$ is the largest radical such that $\gamma(R) \cap \beta(R) \subseteq \alpha(R)$ for every ring $R$.

Proof. I. Let $R / I$ and $I$ be in $\mathscr{R} . \alpha(I)$ is an ideal of $R[8$, p. 124]. Consider $R / \alpha(I)$.

$$
\beta(R / \alpha(I)) \cap I / \alpha(I)=\beta(I / \alpha(I)) \subseteq \alpha(I / \alpha(I))=0
$$

since $\beta$ is hereditary and $I$ is in $\mathscr{R}$. It follows that

$$
\beta(R / \alpha(I)) \cong \frac{\beta(R / \alpha(I))+I / \alpha(I)}{I / \alpha(I)}
$$

which is an ideal of $R / \alpha(I) / I / \alpha(I) \cong R / I$, which is in $\mathscr{R}$. Hence

$$
\frac{\beta(R / \alpha(I))+I / \alpha(I)}{I / \alpha(I)} \cong \alpha\left(\frac{R / \alpha(I)}{I / \alpha(I)}\right)
$$


$\alpha$ is hereditary, hence $\beta(R / \alpha(I))$ is $\alpha$-radical. Let $K / \alpha(I)=\beta(R / \alpha(I))$. $K$ is an extension of an $\alpha$-radical ring by an $\alpha$-radical ring and hence is $\alpha$-radical.

$$
\frac{\beta(R)+\alpha(I)}{\alpha(I)} \leqq \beta(R / \alpha(I))=K / \alpha(I)
$$

hence $\beta(R) \subseteq \beta(R)+\alpha(I) \subseteq K \cong \alpha(R)$.

If $J$ is any ideal of $R$, then $R / J$ is an extension of $(I+J) / J$ by $R /(I+J) \cong(R / J) /(I+J) / J . \quad \mathscr{R}$ is clearly closed under homomorphic images, hence $I+J / J$ and $R / I+J$ are in $\mathscr{R}$. By the above

$$
\beta(R / J) \subseteq \alpha(R / J) \text {. }
$$

Therefore, $R$ is in $\mathscr{R}$ and $\mathscr{R}$ is closed under extensions.

II. Let $R$ be a ring and $\left\{I_{i}\right\}$ a chain of $\mathscr{R}$-ideals. Let $I=\bigcup I_{i}$. If $J$ is an ideal of $I, I / J=\left(\bigcup I_{i}\right)+J / J \neq \bigcup_{i} I_{i}+J / J$.

$$
\begin{aligned}
\beta(I / J) & =\beta(I / J) \cap\left(\bigcup_{i} I_{i}+J / J\right)=\cup\left(\left(\beta(I / J) \cap I_{i}+J / J\right)\right. \\
& =\cup \beta\left(I_{i}+J / J\right) \leqq \cup \alpha\left(I_{i}+J / J \leqq \alpha(I / J)\right) .
\end{aligned}
$$

Therefore $I$ is in $\mathscr{R}$. By Zorn's lemma, there is a maximal $\mathscr{R}$-ideal $J$ of $R$. If $K$ is another, then $K+J$ is an extension of $J$ by $K+J / J$ and hence is in $\mathscr{R}$. It follows that $K=J$. We have then that every ring $R$ contains a maximum $\mathscr{R}$-ideal. It is now trivial that $\mathscr{R}$ is a radical class.

III. Let $\gamma=\gamma$, For any ring $R$,

$$
\gamma(R) \cap \beta(R)=\beta(\gamma(R)) \leqq \alpha(\gamma(R)) \leqq \alpha(R)
$$

since $\beta$ is hereditary and $\alpha(R)$ is in $\mathscr{R}$. It is clear that $\alpha$ is the largest such radical.

COROLlaRY 27. For hereditary radicals $\alpha$ and $\beta$, the radical class of $(\alpha: \beta)$ is $\mathscr{R}=\{R$ : If $I$ is an accessible subring of $R$ and $J$ an ideal of $I, \beta(I / J) \subseteq \alpha(I / J)\}$

Proof. Propositions 24 and 26.

6. Complements. In the previous section, we characterized pseudocomplements in the lattice of hereditary radicals. In this section, we give some partial results as to which of these pseudocomplements are actually complements. If $\alpha$ and $\beta$ are complements, then any ring $R$ is a subdirect sum of an $\alpha$-semisimple ring and a $\beta$-semisimple ring and $\alpha(R)+\beta(R)$ is a large ideal of $R$. Complementary radicals then give a nice decomposition of rings. 
Suppose that $\alpha$ is a complemented hereditary radical. Its complement is its pseudocomplement $\alpha^{*} . \alpha^{* *}$ must be the complement of $\alpha^{*}$, hence we have $\alpha^{* *}=\alpha$. If $Z$ is the zero ring of the integers, either $\alpha(Z)=Z$ or $\alpha^{*}(Z)=Z$, hence either $\alpha$ or $\alpha^{*}$ is hypernilpotent. In this section, we shall always assume $\alpha$ is hypernilpotent and $\alpha^{*}$ is subidempotent.

It is clear that if $\mathscr{S}$ is a radical semisimple class, $\alpha$, (the radical associated with the semisimple class $\mathscr{P}$ ) is complemented. Stewart [19] has characterized such classes. A class $\mathscr{S}$ is a radical semisimple class if and only if there is a positive integer $n$, such that a ring $R$ is in $\mathscr{S}$ if and only if $x^{n}=x$ for every $x$ in $R$. We enlarge this class of complemented radicals with.

Ppoposition 28. The upper radical determined by a finite collection finite fields is complemented.

Proof. Let $\mathscr{F}$ be a finite collection of finite fields and let $\alpha=\mathscr{C}(\mathscr{F}) . \quad \alpha(R)=\cap\{I$ ideal of $R: R / I$ in $\mathscr{F}\}$. By [2, p. 195], $\alpha^{*}(R)=\alpha^{\prime}(R)=\cap\{I$ ideal of $R: R / I$ is subdirectly irreducible with $\alpha$-radical heart\}. Now $\alpha \wedge \alpha^{*}=0$, hence it suffices to show $\alpha \vee \alpha^{*}=1$. Suppose to the contrary that $\alpha \vee \alpha^{*} \neq 1$, then there is a nonzero ring $R$ with $\alpha(R)=\alpha^{*}(R)=0 . \quad R$ is a subdirect sum of rings in $\mathscr{F}$ and a subdirect sum of subdirectly irreducible ring with $\alpha$-radical hearts. Since $|\mathscr{F}|$ is finite, there is an integer such that $x^{n}=x$ for every $x$ in every ring of $\pi$. Therefore $x^{n}=x$ for every $x$ in $R$. If $G$ is a subdirectly irreducible factor ring of $R$, then $G$ must inherit this property and hence $G$ must be commutative [10] with non-nilpotent heart. Hence $G$ is a finite field. Let $n$ be the smallest integer greater than 1 such that $x^{n}=x$ for every $x$ in $R$. Clerly $|G|$ divides $n$. If $G$ is $\alpha$-radical, then $|G|<n$, otherwise $G$ would be in $\mathscr{F}$. If $R$ is a subdirect sum of $\alpha$-radical fields $\left\{G_{i}\right\}$ with $\left|G_{i}\right|$ dividing $n$ for each $i$, it is clear that the least common multiple $d$ of the $\left|G_{i}\right|$ must divide $n$ and $d<n$. Hence $x^{d}=x$ for every $x$ in $R$, a contradiction. Hence $\left(\alpha \vee \alpha^{*}\right)(R) \neq 0$.

Corollary 29. A ring $R$ cannot be a subdirect sum of each of two finite disjoint collections of finite fields.

Corollary 29 is in contrast to the example given in $\S 2$ of a ring which was the subdirect sum of each of two disjoint infinite collections of fields. Note that the fields in that example could be finite by choosing $F_{i}=G F\left(p^{2^{2}}\right)$.

Since $\alpha=\alpha^{* *}$, we have by [2] that $\alpha$ is special. In fact $\alpha$ has 
a special class consisting of subdirectly irreducible rings. Since $\alpha$ is hereditary, a subdirectly irreducible ring will be semisimple if and only if its heart is semisimple; hence, $\alpha$ is completely determined by the simple $\alpha$-semisimple rings.

Let $R$ be a ring. We recall that the centroid of $R$ consists of all endomorphisms of $(R,+)$ that commute with both left and right multiplication by elements of $R$. Toward characterization of $\alpha$-semisimple simple rings, we have

Proposition 30. Let $R$ be an a-semisimple simple ring with $R^{2} \neq 0$. If $\alpha$ is a complemented hereditary radical, then the centroid $C$ of $R$ is finite.

Proof. Suppose to the contrary that $C$ is infinite. Since $R$ is simple, $C$ is a field [11]. $R$ can be regarded as an algebra over $C$. Let $C[x]$ denote the ring of polynomials over $C$ and $(x-a)$ the ideal of $C[x]$ generated by $x-a$. Let $p_{a}$ denote the natural map of $C[x]$ onto $C[x] /(x-a)$. Let $\left\{y_{i}\right\}$ be a basis of $R$ over $C . R[x]$ is isomorphic to the tensor product $R \bigotimes_{c} C[x]$. Consider the map

$$
1 \otimes p_{i}: R \bigotimes_{c} C[x] \rightarrow R \bigotimes_{c} C\left[x \mid /(x-a) \cong R \bigotimes_{c} C \cong R\right.
$$

$1 \otimes p_{a}$ is onto. Every element of $R \otimes_{c} C[x]$ can be written uniquely as $\sum_{i} y_{\imath} \otimes q_{\imath}(x)$. This is mapped to $\sum_{i} y_{i} \otimes\left(q_{i}(x)+(x-a)\right)$. This is 0 if and only if $q_{i}(x)$ is in $(x-a)$ for each $i$. Now $\bigcap_{a} \operatorname{ker}\left(1 \otimes p_{a}\right)=0$ since $C$ is infinite. $R[x]$ then is a subdirect sum of copies of $R$ and hence is $\alpha$-semisimple. Let $I=\alpha^{*}(R[x]) . \quad I \neq 0$ since $\alpha \vee \alpha^{*}=1$. $x I$ is a nonzero ideal of $I$ so $\alpha^{*}(x I)=x I$. Clearly $(x I)^{2} \neq x I$. $\alpha^{*}$ is subidempotent hence $x I$ is $f$-regular which implies $(x I)^{2}=x I[7]$, a contradiction.

There clearly remains a gap between Proposition 30 and Proposition 28 which we have been unable to close.

\section{REFERENCES}

1. T. Anderson, N. Divinsky, and A. Sulinski, Hereditary radicals in associative and alternative rings, Canad. J. Math., 17 (1965), 594-603.

2. V. A. Andrunakievic, Radicals in associative rings, I, Mat. Sbornik (86) 44 (1958), 179-212 (Russian).

3. E. P. Armendariz, Closure properties in radical theory, Pacific J. Math., 26 (1968), $1-7$.

4. Hereditary subradicals of the lower Baer radical, Publ. Math. Debrecen., 15 (1968), 91-93.

5. E. P. Armendariz and W. G. Leavitt, Nonhereditary semisimple classes, Proc. Amer. Math. Soc., 18 (1967), 1114-1117.

6. Garrett Birkhoff, Lattice theory, 3rd Edition, American Mathematical Society, Providence, R. I., 1967. 
7. R. L. Blair, $A$ note on f-regularity in rings, Proc. Amer. Math. Soc., 6 (1955), 511-515.

8. N. J. Divinsky, Rings and Radicals, Univ. of Toronto Press, Toronto, 1965.

9. A. E. Hoffman and W. G. Leavitt, Properties inherited by the lower radical, Portugal Math., (To appear).

10. N. Jacobson, Structure theory of algebraic algebras of bounded degree, Ann. of Math., 46 (1945) 695-707.

11. - Structure of rings, American Mathematical Society, Providence, R. I., 1964. 12. A. G. Kurosh, Radicals of rings and algebras, Mat. Sbornik (75) 33, 13-26 (Russian). 13. W. G. Leavitt, Sets of radical classes, Publ. Math. Debrecen, 14 (1967), 321-324.

14. - Radical and semisimple classes with specified properties, Proc. Amer. Math. Soc., 24 (1970), 680-687.

15. Yu-lee and R. E. Propes, On intersections and union of radical classes, Notices Amer. Math. Soc., 17 (1970), 235.

16. G. Michler, Radikale und Sockel, Math. Ann., 167 (1966) 1-48.

17. Gary L. Musser, On linear semiprime ( $p ; q)$ radicals, Pacific J. Math., (To appear).

18. Ju. M. Rjabuhin, Overnilpotent and special radicals, Studies in Algebra and Math. Anal., 65-72. Izdat. "Karta Moldovenjaske," Kishinev, 1965 (Russian).

19. P. N. Stewart, Semi-simple radical classes, Pacific J. Math., 32 (1970), 249-254.

Received November 5, 1970 and in revised form August 19, 1971.

UNIVERSITy OF Miami 


\section{PACIFIC JOURNAL OF MATHEMATICS}

\section{EDITORS}

H. SAMELSON

Stanford University

Stanford, California 94305

C. R. HOBBY

University of Washington

Seattle, Washington 98105
J. DugundJI

Department of Mathematics

University of Southern California

Los Angeles, California 90007

RICHARD ARENS

University of California

Los Angeles, California 90024

\section{ASSOCIATE EDITORS}

E. F. BeCKENBACH

B. H. NeumanN

F. WOLF

K. YosHIDA

\section{SUPPORTING INSTITUTIONS}

UNIVERSITY OF BRITISH COLUMBIA

CALIFORNIA INSTITUTE OF TECHNOLOGY

UNIVERSITY OF CALIFORNIA

MONTANA STATE UNIVERSITY

UNIVERSITY OF NEVADA

NEW MEXICO STATE UNIVERSITY

OREGON STATE UNIVERSITY

UNIVERSITY OF OREGON

OSARA UNIVERSITY
UNIVERSITY OF SOUTHERN CALIFORNIA STANFORD UNIVERSITY

UNIVERSITY OF TOKYO

UNIVERSITY OF UTAH

WASHINGTON STATE UNIVERSITY

UNIVERSITY OF WASHINGTON

AMERICAN MATHEMATICAL SOCIETY

NAVAL WEAPONS CENTER

Printed in Japan by International Academic Printing Co., Ltd., Tokyo, Japan 


\section{Pacific Journal of Mathematics}

\section{Vol. 40, No. $1 \quad$ September, 1972}

Alex Bacopoulos and Athanassios G. Kartsatos, On polynomials

approximating the solutions of nonlinear differential equations........

Monte Boisen and Max Dean Larsen, Prüfer and valuation rings with zero

divisors ..........................................

James J. Bowe, Neat homomorphisms

David W. Boyd and Hershy Kisilevsky, The Diophantine equation

$$
u(u+1)(u+2)(u+3)=v(v+1)(v+2) \ldots \ldots \ldots \ldots \ldots \ldots \ldots
$$

George Ulrich Brauer, Summability and Fourier analysis ...............

Robin B. S. Brooks, On removing coincidences of two maps when only one,

rather than both, of them may be deformed by a homotopy ............

Frank Castagna and Geert Caleb Ernst Prins, Every generalized Petersen

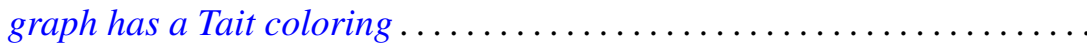

Micheal Neal Dyer, Rational homology and Whitehead products ..........

John Fuelberth and Mark Lawrence Teply, The singular submodule of a

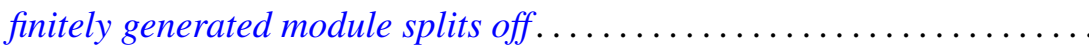

Robert Gold, $\Gamma$-extensions of imaginary quadratic fields ............ 83

Myron Goldberg and John W. Moon, Cycles in k-strong tournaments.......

Darald Joe Hartfiel and J. W. Spellmann, Diagonal similarity of irreducible

matrices to row stochastic matrices...............

Wayland M. Hubbart, Some results on blocks over local fields ..

Alan Loeb Kostinsky, Projective lattices and bounded homomorphisms....

Kenneth O. Leland, Maximum modulus theorems for algebras of operator

valued functions ...

Jerome Irving Malitz and William Nelson Reinhardt, Maximal models in the

language with quantifier "there exist uncountably many" ..

John Douglas Moore, Isometric immersions of space forms in space

forms.

Ronald C. Mullin and Ralph Gordon Stanton, A map-theoretic approach to

Davenport-Schinzel sequences ....................

Chull Park, On Fredholm transformations in Yeh-Wiener space. .

Stanley Poreda, Complex Chebyshev alterations ..............

Ray C. Shiflett, Extreme Markov operators and the orbits of Ryff. ...

Robert L. Snider, Lattices of radicals .....................

Ralph Richard Summerhill, Unknotting cones in the topological

category ................................

Charles Irvin Vinsonhaler, A note on two generalizations of $\mathrm{QF}-3 \ldots \ldots 229$

William Patterson Wardlaw, Defining relations for certain integrally

parameterized Chevalley groups...................

William Jennings Wickless, Abelian groups which admit only nilpotent

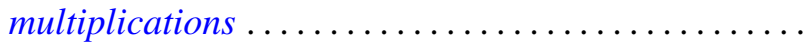

